$\underline{\underline{p}}=\mathrm{O}$

\title{
Mobile based application for prediction of diabetes mellitus: FHIR Standard
}

\author{
Meenakshi Sharma $^{1 *}$ Himanshu Aggarwal $^{2}$ \\ ${ }^{1}$ Computer Science and Engineering Department, G.I.M.E.T,Amritsar, 143501, India; \\ ${ }^{2}$ Computer Engineering Department, Punjabi University, Patiala 147002, India; \\ *Corresponding author E-mail: sharma.minaxi@gmail.com
}

\begin{abstract}
Presently physicians providing clinical decision along clinical guidelines manually on basis of manual query to patients that involve documentation files of lab reports, medication description etc., that make practice sluggish and arduous. With development of mobile based application provide assistance to physician in providing quick decision in appropriate manner as patient data available any-time from any-where that will enhance quality of care in field of healthcare. HL7 standard promotes FHIR(Fast Health Interoperability Resource) standard and health information technology for mobile-health. Mobile health is not a vertical field, but a kind of horizontal field that has cut various impact of healthcare domains. This application provide the effective health management of patient's electronic health record with ease of user friendly interface, that can be accessed on role based authentication by numbers of actors like patient, physician, clinic staff and patient's family etc. Basically this study projected decision support system for prediction of chronic disease-DiabeticMellitus using FHIR(Fast Health Interoperability Resource) as standard for interoperability among hospital information system. Application provide physician's structured series of symptoms/signs as uploaded by patients, laboratory and staff. Series of questioner along with outcomes are formed, all outcomes appraised and tested by team of medical domain experts.
\end{abstract}

Keywords: Access Control; Clinical Decision Support System; Chronic Disease; Electronic Health Records; Mobile Application.

\section{Introduction}

With ease of speedy access to patient health data for healthcare services improve quality of service along with provide safety for survival of patient by accessing data at timely during medical emergencies. With advancement of technology like cloud computing that provide provision of access of patient data at anytime from anywhere. Electronic gadgets like mobiles, PDA, tab are one of important constituent of their daily routine life. Mobile devices now a day's in hub of healthcare domain. Integrated mobile technologies with healthcare process projecting novel emerging model where patient's plays central role in getting concerned healthcare guidelines[1]. With facility of download apps of health related patients get assistance and awareness about various healthcare amenities available in mode of real-time. Adoption rate of mobile based apps among physician has been increased, as they become more responsive and knowledgeable regarding patients necessities and endow with services whenever required. Patient's can utilized mobile based application for their medical urgency. Significantly, mobile e-healthcare application will reduced occupancy of hospitals only with higher emergency will need to visited to hospitals. Mobile technology based CDSS will provide assistant to physician in making their practices and recommendations more effective manner without any misinterpretation along ease of communication with patients and clinic staff. For this study, we have proposed "DIBIT" app for healthcare. DIBIT app provide assistance to patients in finding doctor's specialized in diabetics in their zone and fixed appointments for consultancy.

Diabetes incidence is growing very rapidly and is one of leading cause of mortality globally. Prediction of diabetes at early stage can prevent patient from onset suffering and complication by adapting the preventive countermeasure like changes daily routine. Worldwide ratio of diabetics patients is more than four hundred million and more than $47.3 \%$ are under diagnosis that records itself a alarming situation. As per survey, every tenth person is suffering from this chronic disease, although number of person didn't aware about that they are suffering from this disease. Major problem facing while hospitalization of patient is to access the healthcare services. Unavailability of patient information along with clinical recommendations at right time lead to inappropriate diagnosis of the disease along with cause of hospitalization.

Clinical guidelines information must available in documented form, for easy access on based of evidence statement. Major objective of this application to provide assistance to providers, stakeholders, clinical staff, beneficiaries for their suggestions and interventions. Computer based formulization of such kind of guidelines named as Clinical-interpretable Guidelines(CIG) and provide provision to prepare Clinical decision support system to assist to make decision as compare to written guidelines based assistance. Ontology based model can be used to integrate clinical guidelines with decision support system, which reusability of knowledge base is easier along with ease of standardization issues. An FHIR(Fast Health Interoperatibilty Resource ) based prototype for mobiles using clinical guidelines for diagnosis of diabetics 
mellitus ,presented Through this paper, authors provide insight about FHIR usage for mobile based healthcare application. Interface like REST API's utilized with Android based devices for accessing healthcare application. Security aspects also be considered for patients information. Rest of paper has been organized into various sections as follows: Framework model utilized for mobile, methodology and security aspects discussed in section two; in section three conclusion and future work to be discussed.

\section{DIBIT mobile application for diabetic melli- tus}

DibIT app whose main objective to induce secure communication between physician and patients to enhance patient care outcomes by make available of interactive software based tools to exchange healthcare information so that diagnosis can be provide speedily. DibIT provide provision to patients to view specifics about doctor like qualification along experience in practicing, address, consultancy charges and timing's, exact location etc.

With support of such kind of applications, numerous medical oriented tips can adds value in routine life of users. Integration of technology with healthcare domain present new field of research for researchers, vendors and technocrats etc, which can support number of policies with reducing medical cost.. As per report of Department Health of Sao Paulo, information technology in recent years be indispensible element of healthcare system. Appropriate convention of technology in healthcare sector shatter the barrier of monetary resources that supposed to be one of major barrier in healthcare domain world widely. Many factors like massive diagnosis cost along with treatment have great impact on annual balance budget of health care. As per author, steady research on constraint to reducing cost by escalating efficiency while in case of healthcare domain its matter of continued existence.

To overcome barriers of cost and to improve patient care outcomes, mobile based application has been developed with assist of technology of mobile phones [2,14]. DibIT used information like diagnosis ,prediction and observation of ICT (information and communication technologies) for end users. GOE(Global observatory-for e-health) society consider m-health as component for ehealth. Wireless sensors or with support of Bluetooth patient information can be monitors or uploaded to mobile devices[2,15] WHO(World health organization) accountable for globally health oriented concern, policies, standard to be preferred interoperability along research oriented consideration has introduced concepts of e-health like progression, impact of technology to handle health issues through association of GOE[3,4]. Diabetes occurs due to metabolic disorder, is a complex, chronic illness that characterized due to high levels of sugar in the blood of individual body and it originates when the pancreas are inefficient in producing enough insulin, using insulin or both. Variation of the insulin level vary according to the glucose level present in the blood. The blood glucose level get increased when the human body inefficient to generate the adequate or usage of insulin which can leads towards the adverse health complications like damage of Kidneys; heart failure and stroke; amputations of feet ; vision blindness ; hypertension ; early death etc. Broadly the diabetes are classified into three clinical categories Type-1diabetes previously also known as IDDM(Insulin dependent diabetes ,juvenile or childhood-onset), Type-2 diabetes formerly as NIDDM(Non Insulin dependent diabetes or adult-onset) and Gestational diabetes mellitus (GDM). Type 1 diabetes mellitus which arises due to the destruction of beta-cells in body which leads to deficiency of insulin, therefore for the survival patient requires to inject the insulin. Main symptoms of type 1 diabetes are sudden weight loss, polyuria(excessive urine problem), polydipsia(Excessive thirst), tiredness and constant hunger etc. Type 2 class of diabetes results due to resistance of insulin i.e the body can't properly utilize the produced insulin. Type 2 diabetes more prevalent in adults, with the presence of ketoacidosis but now a day's also found in children . Gestational diabetes mellitus, in which the blood glucose level of the body above normal known as hyperglycaemia , is not clearly diabetes but diagnosed in pregnant women which leads to type 2 diabetes in later stage[9].Diabetes has been recognized as debilitating ,complex and chronic disease by WHO(World Health Organization)[5]. Worldwide the estimated diabetics patient of type 2 are 382 million, around 90-95\% cases among both men, women and children. Its $3.3 \%$ of the entire inhabitants. Globally, diabetes is rated as one of the cause of death among top ten diseases, around 1.4 millions death arises due to diabetes. By year,2035 the graph of diabetic patients will drastically increase and around 492 million people will effected by diabetes The stem of our motivation for developing a novel clinical knowledge based reporting system, is the higher prevalence rate of diabetes and lack of efficient such application system for predicting of diabetes at right stage.DibIT application used HL7-FHIR[6,7] standard to provide interoperability among HIS(Hospital information system) and patients to access patient history information. Framework of FHIR[8] shown in Fig.1.

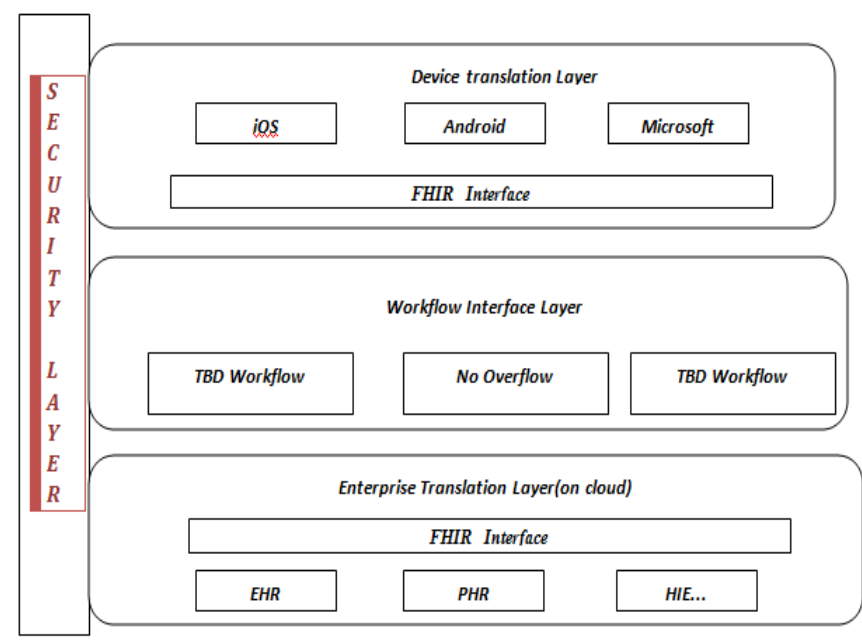

Fig. 1: FHIR framework

Application provide flexibility that patients can monitors and get recommendation associated to their health issues, also minimizing medical errors by receiving online physicians support. Initially, doctor and patients have to registered with application and will receive unique identification number, which used for further communication. Authentication of doctors authenticated by admin only. Entire patients and doctor information stored on cloud in FHIR format along with its resources. RESTful API's used to provide interface among them.

\subsection{Individual health emblem:}

It's the central part of application around which entire concepts of DIBIT evolves, it holds various tab which supports CRUDS (create, read, update, delete and store) operation of FHIR resources related to patients. Resources of FHIR's $[9,10]$ are as follows:

(i) Personal health details: This resource contain personal information about patient. It consist various fields like name, age, gender, address for correspondence, photograph, UID(adhar number) and details about insurance policies along with policy number. (ii) Health oriented details: This represent about general details related to the patient. It includes patient's family history, allergic issues, detail about any injury, surgery, about other chronic disease and health related complications.

(iii) Laboratory and Diagnosis Details: Provide the listing about past diagnosis analysis as well as about laboratory examination reports. Also hold current report of the lab test to be performed along with results.

(iv) Medication details: Provide information about medication diet plan prescribed by physician. Also provide details regarding intake schedule, timings of medication. 
(v) Plan details: Physician design care plan that patient has to follow along with actions.

Pending details of planned that patient still follow afterwards shown in different color pattern so that subject can notify plan very easily.

Preliminary step to make use of application, user need to register himself and must have authentic user password to get assistance from application in some specific order as per requirement. It's a role based application, where actor play role which has been assigned like only a physician can make diagnosis of newly registered patient, medication prescription details and diet, care plan etc..All kind of conversation store and retrieve from FHIR based servers in form of resources for future communication. Patient can upload directly vital statics for continuous monitoring of health issues for example in care-diet plan can provide information regarding calories burnt during exercise, strictly follow diet chart etc.. With relevant to medical-prescription either intake medicine timely or not.

\subsection{Security- aspects of DIBIT}

To deal with security challenges of mobile based DIBIT application, author implemented mechanism of security at diverse levels and provide protection to clinical patient data preferred role based access mechanism[11,12] For application two kind of roles has been created which are as : (i) Owner based role (ii) Trusted party based role. Patient has been designated with owner role, full access to his/her data i.e. patient has various privileges to perform CRUDS operation, while trusted party can be doctor, clinical staff anonymous and family friend of patient. Different privileges are provided to different actor as shown in table 1.Here in anonymous role can be referred by government, researchers for making any kind of survey, research analysis or invention of new medicine.

Table 1: Role based policies to access health information

\begin{tabular}{|c|c|c|c|c|c|c|c|c|c|c|c|c|c|c|c|c|c|c|}
\hline \multirow[t]{2}{*}{ Role } & \multicolumn{3}{|c|}{$\begin{array}{c}\text { Personal } \\
\text { Information }\end{array}$} & \multicolumn{3}{|c|}{$\begin{array}{c}\text { General } \\
\text { Health- } \\
\text { Information }\end{array}$} & \multicolumn{4}{|c|}{$\begin{array}{c}\text { Diagnosis } \\
\text { results }\end{array}$} & \multicolumn{4}{|c|}{$\begin{array}{l}\text { Laboratory-test } \\
\text { reports }\end{array}$} & \multicolumn{4}{|c|}{$\begin{array}{l}\text { Diet-Care } \\
\text { Plan }\end{array}$} \\
\hline & $C R$ & $U$ & $D$ & $d R$ & $U$ & $D$ & & & $U$ & $D$ & $C$ & $R$ & $\bar{U}$ & $D$ & ( & & $\bar{U}$ & $D$ \\
\hline Patient & $\sqrt{1}$ & $\sqrt{ }$ & $\sqrt{ }$ & $\sqrt{ }$ & & & & & & & & $\sqrt{ }$ & & & & & & \\
\hline Physician & $\sqrt{ }$ & & & $\sqrt{ }$ & & & & & $\sqrt{ }$ & & & $\sqrt{ }$ & & & 1 & & $\sqrt{ }$ & \\
\hline $\begin{array}{l}\text { Clinical } \\
\text { Staff }\end{array}$ & $\sqrt{ }$ & & & $\sqrt{ }$ & & & & & & & & $\sqrt{ }$ & & & & & & \\
\hline $\begin{array}{l}\text { Anonymo } \\
\text { us }\end{array}$ & $\sqrt{ }$ & & & $\sqrt{ }$ & & & & & & & & $\sqrt{ }$ & & & & & & \\
\hline
\end{tabular}

Access to user also categorized into two kind : (i) short period access (ii) long term access. Every time user when make access to application, receive an otp on basis of short or long term duration period. Short access provided when patient interact with pharmacists regarding medicine prescribed by physician $[13,14]$. Report of provided medication automatically received to physician also with detailed of patient and its unique id along with photograph for clarity. While in long term login process scenario, patient visit hospital, have diagnosis by concerned physician and perform test as recommended by physician. All information in form of list stored on server side and accessible to trusted party as well as by owner with provided privileges. As security aspect in application patient and other actors don't have privileges to make any amendments to electronic health data stored on server. Only short term access issued to read patient data. While access mode active, all records of log maintained with time duration so that on any excruciating access accountability can be initiated. Access to user also categorized into two kind : (i) short period access (ii) long term access . Every time user when make access to application, receive an otp on basis of short or long term duration period. Short access provided when patient interact with pharmacists regarding medicine prescribed by physician[13,14]. Report of provided medication automatically received to physician also with detailed of patient and its unique id along with photograph for clarity. While in long term login process scenario, patient visit hospital, have diagnosis by concerned physician and perform test as recommended by physician. All information in form of list stored on server side and accessible to trusted party as well as by owner with provided privileges. As security aspec $t$ in application patient and other actors don't have privileges to make any amendments to electronic health data stored on server. Only short term access issued to read patient data. While access mode active, all records of log maintained with time duration so that on any excruciating access accountability can be initiated[16].

\section{Methodology}

Numerous mobile device held the capability to confine health oriented information and events. Data collected from heterogeneous devices could have data in different format, that create barrier to exchange data among HIS and other devices. FHIR standard API alleviate provision to establishment of interoperable for mobile based applications. Legacy standards of Healthleavel-7 like $\mathrm{v}-1, \mathrm{v}-2$ etc. has been introduced, but due to some limitations FHIR standard has been introduced to provide interoperability. Adoption rate of FHIR quite more as compared to legacy version by domain of healthcare.

FHIR a kind lightweight scalable standard whose implementation cost economical and set of resources that can be customized

RESTful API's used to provide interface among HIS(hospital information system) with support of CRUD function. DIBIT mobile based application utilized client-server model to perform communication and through RESTful interface resources of FHIR can be exchanged. This application developed under Android environment and been tested on various electronic gadgets. Server based on J2EE platform whereas library interface like JSON/XML used to exchange messages both on server and client side. Attractive compatibility of FHIR standard provide connivance to develop user friendly mobile application for healthcare domain. DIBIT work in client server model, where on client part CRUD operation can be performed with interface of REST API which bound operation to specific UI resources that leads to various calls of AJAX which executing in the background of Android platform. Every electronic gadgets precincts on power supply backup , bandwidth and processing speed. DIBIT application conserve mode of responsiveness during busy in fetching demanded resources.

Another this application also keep record of number of resources utilization along with aggregation. All calls related to control policies of REST interface handled with support of filtering technique based on HTTP. FHIR resources and associated relation of communication are mapped to relational database i.e NoSQL that create repository that can be accessed at any time.

\section{Conclusion}

Through this study, mobile based application named "DibIT" presented that has provide support of interoperability among various actors and HIS (Health information system) using emerging HL7-FHIR standard of healthcare, which assist in implementation and accessibility of mobile based application suitable in domain of healthcare hub with progression of apposite technologies. The DibIT mobile based application accessed through Android ,Microsoft and iOS , based on FHIR standard for real time monitoring of healthcare by providing the provision of exchanging information among patient's, physician, clinic staff etc.. Entire information stored in repository in form of resources. With advancement of technology like IoT, wearable body sensors etc. provide wider spectrum in adoption of mobile based applications. In future, integrate DibIT with rear-time monitoring body weara- 
ble devices like speedometer, glucose meter, pulse meter etc..Also enhance security aspects models of healthcare domain, role based access enhanced to attribute based model for superior grained access mechanism to patient health folder. Encryption techniques like CP-ABE(Ciphertext-Policy Attribute-Based Encryption)applied during exchanging and storing information on server and client end as well as on personal health folder.

\section{Acknowledgement}

The corresponding author indebted to Dr. Sandeep Sharma(Prof. \& Head GNDU) and Dr. Raman Sharma(Prof. Govt. Medical College) for their throughout guidance and motivation in my research journey.

\section{References}

[1] C. Bertolini, Z. Liu, M. Schaf, and V.Stolz. "Towards a Formal Integrated Model of Collaborative .Healthcare Workflows", Foundations of Health Informatics Engineering and Systems, Vol.7151,No.7(2012) pp. 5774.https://doi.org/10.1007/978-3- 642- 32355-3_4,last visit on 10.01.2018.

[2] B.Furht,Ankur Agarwal.."Mobile Medical and Healthcare Appli cations" Handbook of Medical and Healthcare Technologies, pp. 3-15,2013, https://doi.org/10.1007/978-1-46148495-0,last visit on 8. 12.2017.

[3] World Health Organization. MHealth: New horizons for health through mobile technologies, Global observatory for eHealth series -V.3 (2011). last visit on 8. 12.2017

[4] GOE. Global Observatory for eHealth (2014). http://www.who.int/goe/en/, last visit on 8. 12.2017.

[5] WHO, WorldHealthOrganization(2014). http://www.who.int/about/en/, last visit on 15-12-2017.

[6] HL7. http://http://www.hl7.org/, 2016 http://hl7.org/implement/standards/fhir/, last visit on 16-012018.

[7] D. Bender, and K. Sartipi,"HL7 FHIR: An Agile and RESTful ap proach to healthcare information exchange" in IEEE 26th International Symposium on Computer-Based Medical Systems (CBMS), pp. 326-331, 2013

[8] Kim HS \& Jeong HS (2007), A nurse short message service by cellular phone in type-2 diabetic patients for six months. Journal of Clinical Nursing 16, 1082-1087.

[9] Meenakshi sharma and Dr. Himanshu Aggarwal,"EHR Adoption in India: Potential and the Challenges", Indian Journal of Science and Technology, 2016. Vol. 09, No.34 (2016),pp.5762 https://doi.org10.17485/ijst/2016/v9i34/ 100211.

[10] T. H. Y, P.H. Cheng, C.H. Yang, F. Lai, C.L.Chen, "A scalable multi-tier architecture for the national taiwan university hospital information system based on HL7 standard",In Symposium on Computer-Based Medical Systems, pages 99-104, 2006.

[11] W.V Sujansky ,K. Sartipi.," The development of a highly constrained Health Level 7 implementation guide to facilitate electronic laboratory reporting to ambulatory electronic health record systems",. Journal of the American Medical Informatics Association, 16(3):285-290, 2009.

[12] M. Boulos, S. Wheeler, C. Tavares, and R. Jones," How smartphones are changing the face of mobile and participatory healthcaren overview", Biomedical Engineering online, vol. 10, 2011.

[13] J. Sun, X. Zhu, C. Zhang, and Y. Fang, "Security and Privacy for Mobile Healthcare (m-Health) Systems", in Handbook on Securing Cyber-Physical Infrastructure, S. Das, K. Kant, and N. Zhang, Eds. Amsterdam, The Netherlands: Elsevier, 2011.

[14] Dia Griauzde,Jeffrey T. Kullgren,Brad Liestenfeltz,Caroline Rich ardson and Michele Heisler,"A mobile phone- based program to promote healthy behaviors among adults with prediabetes: study protocol for a pilot randomized controlled trial.", Griauzde et al. Pilot and Feasibility Studies,4:48,2018., https://doi.org/ 10.1186/ s40814-018-0246-z .

[15] Sri Vijay Bharat Peddi1 , Pallavi Kuhad1 , Abdulsalam Yassine1, Parisa Pouladzadeh1 Shervin Shirmohammadi1,2, Ali Asghar Nazari Shirehjini,"An Intelligent Cloud-Based Data Processing Broker for Mobile e-Health Multimedia Applications",Future Gen eration Computer Systems,2016.

[16] Boyi Xu,Lida Xu,,"The design of an m-health monitoring system based on a cloud computing platform", Journal Enterprise Information Systems , June,2015 